\title{
Outer Synchronization between Two Coupled Complex Networks and Its Application in Public Traffic Supernetwork
}

\author{
Wen-ju Du, ${ }^{1}$ Jian-gang Zhang, ${ }^{2}$ Xin-lei An, ${ }^{2}$ Shuang Qin, ${ }^{2}$ and Jian-ning $Y u^{1}$ \\ ${ }^{1}$ School of Traffic and Transportation, Lanzhou Jiaotong University, Lanzhou 730070, China \\ ${ }^{2}$ School of Mathematics and Physics, Lanzhou Jiaotong University, Lanzhou 730070, China \\ Correspondence should be addressed to Wen-ju Du; duwenjuok@126.com
}

Received 13 October 2015; Revised 23 December 2015; Accepted 5 January 2016

Academic Editor: David Arroyo

Copyright (c) 2016 Wen-ju Du et al. This is an open access article distributed under the Creative Commons Attribution License, which permits unrestricted use, distribution, and reproduction in any medium, provided the original work is properly cited.

\begin{abstract}
The paper presents a new urban public traffic supernetwork model by using the existing bus network modeling method, consisting of the conventional bus traffic network and the urban rail traffic network. We investigate the synchronization problem of urban public traffic supernetwork model by using the coupled complex network's outer synchronization theory. Analytical and numerical simulations are given to illustrate the impact of traffic dispatching frequency and traffic lines optimization to the urban public traffic supernetwork balance.
\end{abstract}

\section{Introduction}

In recent years, the study of complex networks and their synchronization has made significant achievements. However, most researches are mainly focused on the complex network's "inner synchronization" and not many on the study of synchronization between two networks. $\mathrm{Li}$ et al. [1] focus on two unidirectionally coupled networks and derive analytically a criterion for the synchronization of these two networks. Tang et al. [2] designed effective adaptive controllers and addressed the theoretical analysis of synchronization between two complex networks with nonidentical topological structures. Chen et al. [3] presented a general network model for two complex networks with time-varying delay coupling and derived a synchronization criterion by using adaptive controllers. Sun et al. [4] investigated the linear generalized synchronization between two complex networks. Wang et al. [5] designed an adaptive controller to achieve synchronization between two different complex networks with time-varying delay coupling. In [6] the outer synchronization between two complex networks with discontinuous coupling is studied and the sufficient conditions for complete outer synchronization and generalized outer synchronization are obtained. In these studies most of authors used the Lyapunov stability theorem to prove the synchronization of the complex networks. It is a fact that many methods used Lyapunov analysis theorem to prove the stability of the closed-loop systems. Liu and Tong [7] considered a class of multi-input multioutput nonlinear systems with unknown functions and unknown dead-zone inputs, and the stability of the closedloop system is proved via the Lyapunov stability theorem. In [8] an adaptive fuzzy optimal control design is addressed for a class of unknown nonlinear discrete-time systems, and they proved the stability of the control systems based on the difference Lyapunov function method.

In real life, there exist a lot of complex systems and these complex systems can be described by complex networks. However, with the expansion of networks size and increasing complex of networks connection, there appear many network systems beyond the general networks. There will be a problem of networks nested in other networks when we study these very large scale network systems. In some cases, we cannot completely describe the network's characteristics in the real world by means of the general network diagram theory. Sheffi [9] and Nagurney and Dong [10] put this kind of network that "above and beyond" existing network referred to as supernetwork. Supernetwork model can be used to describe and express the interaction and influence between networks. 
At the same time, a growing body of research is about the application of complex network theory in the urban traffic system [11-15]. However, most studies only focus on the static characteristics of complex network and its stability, such as the topology properties study of traffic network, the study of reliability and robustness of the network, and the research of structure optimization. There are few literatures that studied the dynamic characteristics of urban public traffic network. An et al. [16] construct the urban traffic network models with multiweights taking different bus lines in bus transfer junction as the network nodes and study the global synchronization of the new network model by changing congestion degrees, transfers coefficient, and passenger flow density between different bus lines. They only considered the conventional bus traffic network; however, as two types of primary mode of transport in urban public transportation system, effective coordination between urban rail traffic and conventional bus traffic can increase the accessibility and flexibility, make them complementary advantages, and improve the transport efficiency of overall transportation system. So, the research which combines the conventional bus traffic network and the urban rail traffic network has more practical significance.

Based on the theory of supernetwork and considering the conventional bus traffic network and the urban rail traffic network, we construct an urban public traffic supernetwork model by using the existing bus network modeling method. The synchronization problem of urban public traffic supernetwork model is studied by using the coupled complex network's outer synchronization theory. More precisely, we designed a controller and make the network system reach steady state. And there are many scholars that studied the tracking problem of complex systems by using the Lyapunov stability criterion. Liu and Tong [17] studied the adaptive tracking control problem for a class of nonlinear discretetime systems with dead-zone input and proved that all the signals in the closed-loop system are semiglobally uniformly ultimately bounded and the tracking error converges to a small neighborhood of zero. An effective adaptive control approach is constructed to stabilize a class of nonlinear discrete-time systems in [18], and they proved the tracking error to be regulated to a small neighborhood around zero. In this paper, a controller is designed and makes the system errors converge to zero. At last, we discussed the impact of two types of public traffic vehicles' departing frequency and the coordinated scheduling in the process of transfer between conventional bus traffic and urban rail traffic to the new urban public traffic supernetwork model's synchronous ability.

The paper is organized as follows. The synchronization theory of two networks with mutual connections is presented in Section 2. In Section 3, a new urban public traffic supernetwork model is established. Simulation results are given to show the impact of traffic dispatching frequency and traffic lines optimization to the urban public traffic supernetwork balance in Section 4. In Section 5, we conclude the paper.

\section{The Synchronization Theory of Two Networks with Mutual Connections}

Consider two networks with mutual connections [19], and they both consisting of $N$ nodes can be described by

$$
\begin{aligned}
\dot{x}_{i}(t)= & g\left(x_{i}(t)\right)+\varepsilon_{1} \sum_{j=1}^{N} a_{i j} x_{j}(t) \\
& +\mu \sum_{j=1}^{N} c_{i j}\left(y_{j}(t)-x_{i}(t)\right), \\
\dot{y}_{i}(t)= & g\left(y_{i}(t)\right)+\varepsilon_{2} \sum_{j=1}^{N} b_{i j} y_{j}(t) \quad(i=1,2, \ldots, N), \\
& +\mu \sum_{j=1}^{N} c_{i j}\left(x_{j}(t)-y_{i}(t)\right)+v_{i}(t), \\
& (i=1,2, \ldots, N),
\end{aligned}
$$

where $v_{i}(t)$ are the feedback controllers. For $t>0, x_{i}=$ $\left(x_{i 1}, x_{i 2}, \ldots, x_{i n}\right)^{\mathrm{T}} \in R^{n}, y_{i}=\left(y_{i 1}, y_{i 2}, \ldots, y_{i n}\right)^{\mathrm{T}} \in R^{n}$ are the state vectors of the $i$ th node in the driving network (1) and the response network (2), respectively. $g(\cdot): R^{n} \rightarrow R^{n}$ is a nonlinear continuous differentiable vector function, and the constants $\varepsilon_{1}, \varepsilon_{2}$ denote the inner coupling strengths of network (1) and network (2), respectively; the constant $\mu$ denotes the outer coupling strength. Coupling matrices $A=$ $\left(a_{i j}\right) \in R^{N \times N}, B=\left(b_{i j}\right) \in R^{N \times N}$ are, respectively, the inner connection matrices of the driving network (1) and response network (2), and $A, B$ are an irreducible matrix with zerosum rows, where $a_{i i}=-\sum_{j=1, i \neq j}^{N} a_{i j}, b_{i i}=-\sum_{j=1, i \neq j}^{N} b_{i j}$, and $a_{i j}\left(b_{i j}\right)$ are defined as follows: if there is a connection from node $j$ to node $i(i \neq j)$, then $a_{i j}\left(b_{i j}\right)=1$; otherwise $a_{i j}\left(b_{i j}\right)=$ $0(i \neq j) . C=\left(c_{i j}\right) \in R^{N \times N}$ is the coupling matrix between two networks, where $c_{i j}$ are defined as follows: if there is a connection from node $i$ (belongs to network (1)) to node $j$ (belongs to network (2)), then $c_{i j}=1$; otherwise $c_{i j}=0$.

Definition 1. Outer synchronization between networks (1) and (2) is said to be achieved, if

$$
\lim _{t \rightarrow \infty}\left\|y_{i}(t)-x_{i}(t)\right\|=0, \quad i=1,2, \ldots, N .
$$

Define the errors vector by $e_{i}(t)=y_{i}(t)-x_{i}(t)$; the goal of the controllers $v_{i}(t)$ is to keep system (1) synchronized; that is, $\lim _{t \rightarrow \infty}\left\|e_{i}(t)\right\|=0(i=1,2, \ldots, N)$. The error system can be described by

$$
\begin{aligned}
\dot{e}_{i}(t)= & \dot{y}_{i}(t)-\dot{x}_{i}(t) \\
= & g\left(y_{i}(t)\right)+\varepsilon_{2} \sum_{j=1}^{N} b_{i j} y_{j}(t) \\
& +\mu \sum_{j=1}^{N} c_{i j}\left(x_{j}(t)-y_{i}(t)\right)-g\left(x_{i}(t)\right)
\end{aligned}
$$




$$
\begin{aligned}
& -\varepsilon_{1} \sum_{j=1}^{N} a_{i j} x_{j}(t)-\mu \sum_{j=1}^{N} c_{i j}\left(y_{j}(t)-x_{i}(t)\right)+v_{i} \\
= & g\left(y_{i}(t)\right)-g\left(x_{i}(t)\right)-\left(\mu \sum_{j=1}^{N} c_{i j}+k\right) e_{i}(t) \\
& +\left(\varepsilon_{1} \sum_{j=1}^{N} a_{i j}+\varepsilon_{2} \sum_{j=1}^{N} b_{i j}-\mu \sum_{j=1}^{N} c_{i j}\right) e_{j}(t) .
\end{aligned}
$$

Assumption 2. If there exists a positive constant $L$ such that

$$
\|g(y)-g(x)\| \leq L\|y-x\|,
$$

then function $g(\cdot)$ is called Lipschitz continuous, where $x$ and $y$ are time-varying vectors.

Lemma 3 (see [20]). Let $x_{0}$ be an equilibrium point for $\dot{x}=$ $f(x)$ and let $D \subset R^{n}$ be a domain containing $x_{0}$. Let $V: D \rightarrow$ $R$ be a continuously differentiable function such that

$$
\begin{gathered}
V\left(x_{0}\right)=0, \\
V(x)>0, \\
\forall x \in D-\left\{x_{0}\right\}, \\
\dot{V}(x) \leq 0, \quad \forall x \in D-\left\{x_{0}\right\} .
\end{gathered}
$$

Then, $x_{0}$ is stable. Moreover, if

$$
\dot{V}(x)<0, \quad \forall x \in D-\left\{x_{0}\right\},
$$

then $x_{0}$ is asymptotically stable, where $\dot{V}(x)=\sum_{i=1}^{N}(\partial V /$ $\left.\partial x_{i}\right) f_{i}(x)$ and $V(x)$ is referred to as Lyapunov function.

Theorem 4. Suppose that Assumption 2 holds. We select the controllers

$$
\begin{aligned}
v_{i}(t)= & \varepsilon_{1} \sum_{j=1}^{N} a_{i j} y_{j}(t)-\varepsilon_{2} \sum_{j=1}^{N} b_{i j} x_{j}(t) \\
& -k\left(y_{i}(t)-x_{i}(t)\right), \quad(i=1,2, \ldots, N) ;
\end{aligned}
$$

then the driving network (1) and the response network (2) can realize outer synchronization under the controllers (8), where $k$ is a positive constant.

\section{A New Urban Public Traffic Supernetwork Model}

We can regard the conventional bus traffic system and the urban rail traffic system as a complex network, which consists of traffic stations and traffic lines; the traffic stations connect with the traffic lines, and traffic lines connect with some traffic stations. From the point of view of traffic stations networks, traffic lines networks, and the traffic transfer networks, one can establish three public traffic network models [21, 22]: (1) public traffic stations networks: using the space $L$ modeling method and taking traffic stations as the network nodes, the two traffic stations have edge if they are adjacent in a traffic line; (2) public traffic transfer networks: using the space $P$ modeling method and taking traffic stations as the network nodes, the two traffic stations have edge if there are direct traffic lines between them; (3) public traffic roads networks: using the space $R$ modeling method and taking traffic lines as the network nodes, the two traffic lines have edge if there are the same traffic stations between them.

This paper established a new public traffic supernetwork model based on the existing modeling methods and the modeling idea as follows: taking traffic stations as the network nodes, there is one edge between two nodes if there are direct traffic routes between two traffic stations, established conventional bus traffic network, and urban rail traffic network, respectively. If there is an opportunity to transfer between conventional bus traffic stations and urban rail traffic stations, we link these two different types of nodes and constitute the coupling edges of supernetwork. The conventional bus traffic network, urban rail traffic network, and its coupling edges form a new urban public traffic supernetwork. For example, we take four urban rail stations and four conventional bus stations at Xi'an Xincheng area as the supernetwork nodes. The four urban rail stations are Beidajie subway station, Wulukou subway station, Sajinqiao subway station, and Anyuanmen subway station, ordinal numbers for 1-4. And the four conventional bus stations are Beidajie bus station, Beimen bus station, Revolution park bus station, and Honghu street bus station, ordinal numbers for 5-8. According to the proposed modeling method, the new urban public traffic supernetwork model is established as illustrated in Figure 1.

\section{Numerical Simulation}

Gao and Song [23] draw the conclusions that passenger flow of urban public traffic fulfills the nonlinear behavior. Suppose that passenger flow of both conventional bus traffic lines and urban rail traffic lines fulfills Lorenz chaotic system, so the nodes dynamical equations can be described by

$$
\begin{aligned}
& {\left[\begin{array}{l}
\dot{x}_{i 1} \\
\dot{x}_{i 2} \\
\dot{x}_{i 3}
\end{array}\right]=\left[\begin{array}{ccc}
-10 & 10 & 0 \\
28 & -1 & 0 \\
0 & 0 & -\frac{8}{3}
\end{array}\right]\left[\begin{array}{l}
x_{i 1} \\
x_{i 2} \\
x_{i 3}
\end{array}\right]+\left[\begin{array}{c}
0 \\
-x_{i 1} x_{i 3} \\
x_{i 1} x_{i 2}
\end{array}\right]} \\
& +\left[\begin{array}{l}
M_{i 1} \\
M_{i 2} \\
M_{i 3}
\end{array}\right], \quad i=1,2,3,4, \\
& {\left[\begin{array}{l}
\dot{y}_{i 1} \\
\dot{y}_{i 2} \\
\dot{y}_{i 3}
\end{array}\right]=\left[\begin{array}{ccc}
-10 & 10 & 0 \\
28 & -1 & 0 \\
0 & 0 & -\frac{8}{3}
\end{array}\right]\left[\begin{array}{l}
y_{i 1} \\
y_{i 2} \\
y_{i 3}
\end{array}\right]+\left[\begin{array}{c}
0 \\
-y_{i 1} y_{i 3} \\
y_{i 1} y_{i 2}
\end{array}\right]} \\
& +\left[\begin{array}{c}
N_{i 1} \\
N_{i 2} \\
N_{i 3}
\end{array}\right]+\left[\begin{array}{c}
v_{i 1} \\
v_{i 2} \\
v_{i 3}
\end{array}\right], \quad i=1,2,3,4,
\end{aligned}
$$




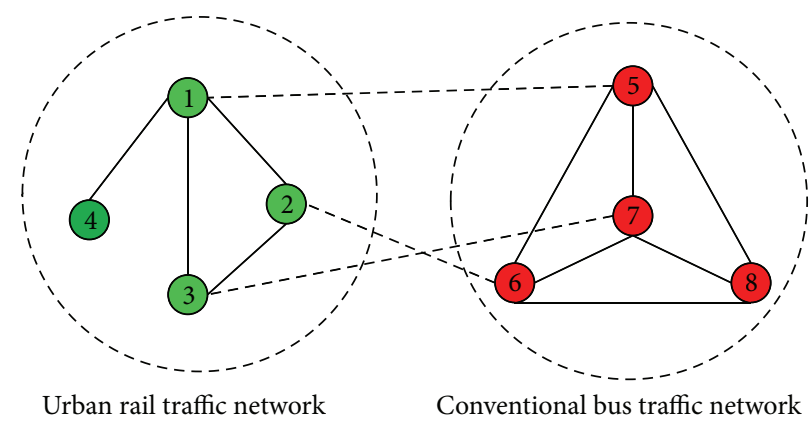

FIGURE 1: The topology map of urban public traffic supernetwork.

where

$$
\begin{aligned}
& M_{i 1}=\varepsilon_{1} \sum_{j=1}^{4} a_{i j} x_{j 1}+\mu \sum_{j=1}^{4} c_{i j}\left(y_{j 1}-x_{i 1}\right), \\
& M_{i 2}=\varepsilon_{1} \sum_{j=1}^{4} a_{i j} x_{j 2}+\mu \sum_{j=1}^{4} c_{i j}\left(y_{j 2}-x_{i 2}\right), \\
& M_{i 3}=\varepsilon_{1} \sum_{j=1}^{4} a_{i j} x_{j 3}+\mu \sum_{j=1}^{4} c_{i j}\left(y_{j 3}-x_{i 3}\right), \\
& N_{i 1}=\varepsilon_{2} \sum_{j=1}^{4} b_{i j} y_{j 1}+\mu \sum_{j=1}^{4} c_{i j}\left(x_{j 1}-y_{i 1}\right), \\
& N_{i 2}=\varepsilon_{2} \sum_{j=1}^{4} b_{i j} y_{j 2}+\mu \sum_{j=1}^{4} c_{i j}\left(x_{j 2}-y_{i 2}\right), \\
& a_{23}=1, \\
& a_{22}=-2, \\
& a_{14}=1, \\
& a_{13}=\varepsilon_{2} \sum_{j=1}^{4} b_{i j} y_{j 3}+\mu \sum_{j=1}^{4} c_{i j}\left(x_{j 3}-y_{i 3}\right), \\
& a_{i 2}=\varepsilon_{j=1}^{4} a_{i j} y_{j 3}-\varepsilon_{2} \sum_{j=1}^{4} b_{i j} x_{j 3}-k\left(y_{i 3}-x_{i 3}\right), \\
& v_{i 1}=\varepsilon_{1} \sum_{j=1}^{4} a_{i j} y_{j 1}-\varepsilon_{2} \sum_{j=1}^{4} b_{i j} x_{j 1}-k\left(y_{i 1}-x_{i 1} x_{j 2}-k\left(y_{i 2}-x_{i 2}\right),\right.
\end{aligned}
$$

$$
\begin{aligned}
& a_{31}=1, \\
& a_{32}=0, \\
& a_{33}=-1, \\
& a_{34}=0, \\
& a_{41}=1, \\
& a_{42}=0, \\
& a_{43}=0, \\
& a_{44}=-1, \\
& b_{11}=-3, \\
& b_{12}=1, \\
& b_{13}=1, \\
& c_{12}=0, \\
& b_{14}=1, \\
& b_{13}=0, \\
& b_{44}=-3, \\
& b_{43}=1, \\
& b_{22}=-3, \\
& b_{31}=1, \\
& b_{23}=1, \\
& b_{24}=1, \\
& b_{31}=1,
\end{aligned}
$$




$$
\begin{aligned}
& c_{14}=0, \\
& c_{21}=0, \\
& c_{22}=1, \\
& c_{23}=0, \\
& c_{24}=0, \\
& c_{31}=0, \\
& c_{32}=0, \\
& c_{33}=1,
\end{aligned}
$$

$$
\begin{aligned}
& c_{34}=0, \\
& c_{41}=0, \\
& c_{42}=0, \\
& c_{43}=0, \\
& c_{44}=0 .
\end{aligned}
$$

For any vectors $x_{i}$ and $y_{i}$ of the Lorenz chaotic system, there exists a positive constant $R$ such that $\left\|x_{i m}\right\| \leq R,\left\|y_{i m}\right\| \leq$ $R(m=1,2,3)$, since the Lorenz chaotic system is bounded in a certain region. Therefore, one has

$$
\begin{aligned}
\left\|f\left(y_{i}\right)-f\left(x_{i}\right)\right\| & =\sqrt{\left(-y_{i 1} y_{i 3}-\left(-x_{i 1} x_{i 3}\right)\right)^{2}+\left(y_{i 1} y_{i 2}-x_{i 1} x_{i 2}\right)^{2}} \\
& =\sqrt{\left(-y_{i 3}\left(y_{i 1}-x_{i 1}\right)-x_{i 1}\left(y_{i 3}-x_{i 3}\right)\right)^{2}+\left(y_{i 2}\left(y_{i 1}-x_{i 1}\right)+x_{i 1}\left(y_{i 2}-x_{i 2}\right)\right)^{2}} \leq \sqrt{2} R\left\|y_{i}-x_{i}\right\|
\end{aligned}
$$

that is, Assumption 2 is satisfied. If we select $L=1, k=8, \varepsilon_{1}=$ $0.3, \varepsilon_{2}=0.4$, and $\mu=0.6$, the values of the equation $L-\mu c_{i}-$ $k+\varepsilon_{1} A+\varepsilon_{2} B-\mu C$ are all negative. In this case, the conditions of Theorem 4 are satisfied, and the driving network (9) and the response network (10) can achieve outer synchronization.
We randomly chose the initial values of the drive systems and the response systems from $(0,1)$; the simulation results are plotted in Figure 2. Figure 2 shows the outer synchronization error,

$$
E(t)=\sqrt{\sum_{i=1}^{N}\left[\left(y_{i 1}(t)-x_{i 1}(t)\right)^{2}+\left(y_{i 2}(t)-x_{i 2}(t)\right)^{2}+\left(y_{i 3}(t)-x_{i 3}(t)\right)^{2}\right]},
$$

between the driving network and the response network. Obviously, the outer synchronization is achieved.

The purpose of this paper is to study the impact of the optimization of control degrees and the intensity of transfer between conventional bus traffic stations and urban rail traffic stations, that is, the impact of inner coupling strength $\varepsilon_{1}$, $\varepsilon_{2}$ and outer coupling strength $\mu$ on the balance of whole complex supernetwork. The meaning of urban public traffic supernetwork balance is that there is a dynamic balance between running vehicles and bus passengers; that is, the running time of public traffic vehicles is closest to preset time (traffic delay is shortest), and meanwhile the retention time of the passengers in traffic stations is the shortest.

We take $k=8, \mu=0.6$ and plot the synchronization errors for the public traffic supernetwork with different value of $\varepsilon_{1}, \varepsilon_{2}$, as shown in Figure 3. From the simulation results, one can find that the public traffic supernetwork achieves balance in 93 time units when $\varepsilon_{1}=\varepsilon_{2}=0.3$, and the public traffic supernetwork achieves balance in 44 time units when $\varepsilon_{1}=$ $\varepsilon_{2}=0.6$. Namely, the greater the value of inner coupling strengths $\varepsilon_{1}, \varepsilon_{2}$, the shorter the time required to balance complex public traffic supernetwork. Accordingly, the inner coupling strengths have a certain influence on the supernetwork's synchronization; that is, increase of the artificial scheduling (appropriately adjust the departing frequency and time) can speed up the public traffic supernetwork's synchronization, and the public traffic supernetwork can reach steady state faster.

Let $k=8, \varepsilon_{1}=\varepsilon_{2}=0.3$ and plot the synchronization errors for the supernetwork with different value of $\mu$, as shown in Figure 4. It is shown that the public traffic supernetwork achieves balance in 81 time units when $\mu=0.3$, and the public traffic supernetwork achieves balance in 36 time units when $\mu=0.8$. That is to say, the greater the value of outer coupling strengths $\mu$, the shorter the time required for balancing complex public traffic supernetwork; this explains that the coordinated scheduling in the process of transfer and optimization of the transfer facilities can speed up the public traffic supernetwork's synchronization.

\section{Conclusions}

In real life, there exist a lot of complex systems which can be described by complex supernetworks. Based on the theory of supernetwork, this paper establishes a new public traffic supernetwork model, and then we study the balance of the public traffic supernetwork. The simulations results reveal that appropriate adjusting of the departing frequency and 


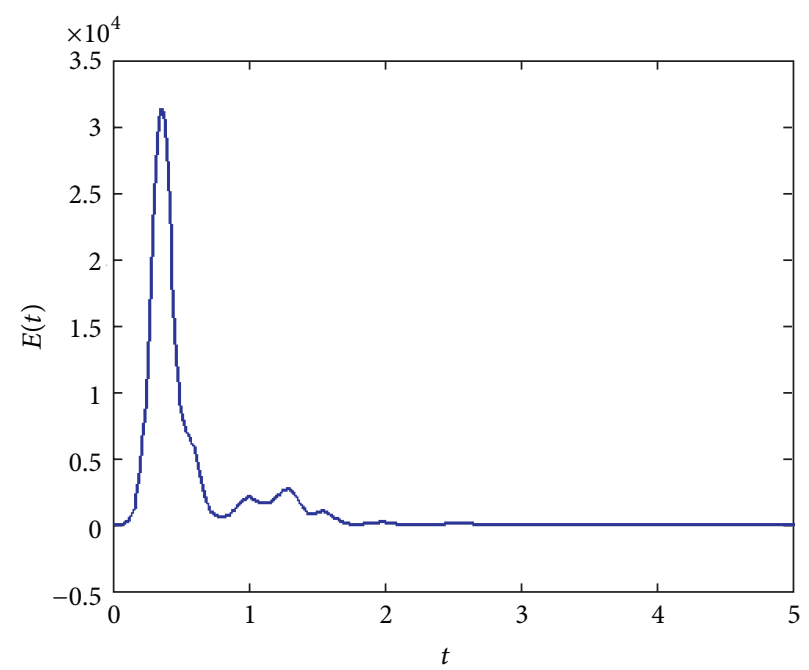

FIGURE 2: The outer synchronization error $E(t)$ between driving network (9) and response network (10).

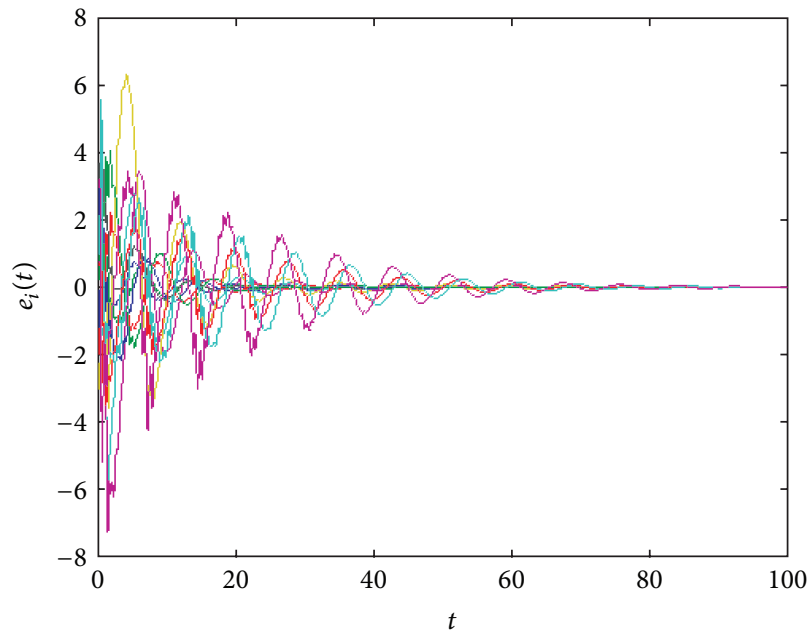

(a) $\varepsilon_{1}=\varepsilon_{2}=0.3$

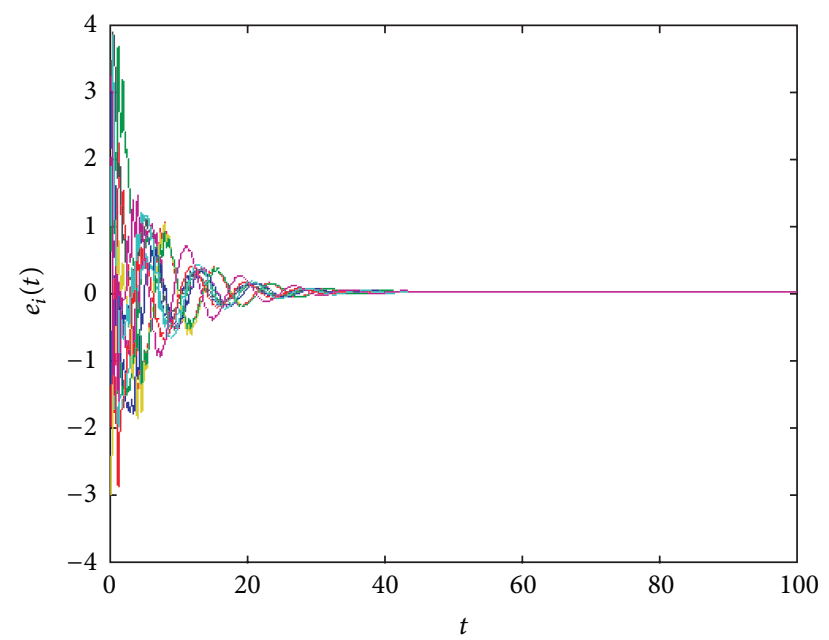

(b) $\varepsilon_{1}=\varepsilon_{2}=0.6$

FIGURE 3: Synchronization errors for the public traffic supernetwork with different value of $\varepsilon_{1}, \varepsilon_{2}$.

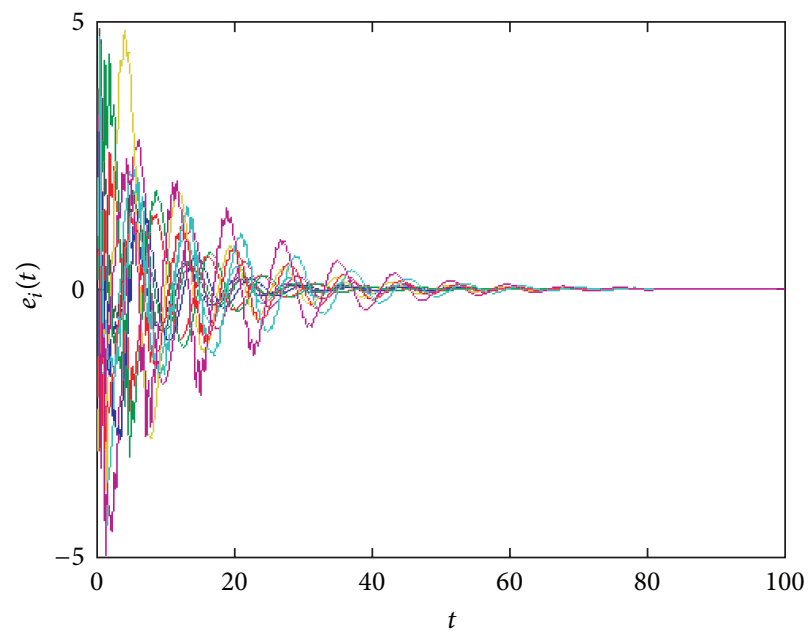

(a) $\mu=0.3$

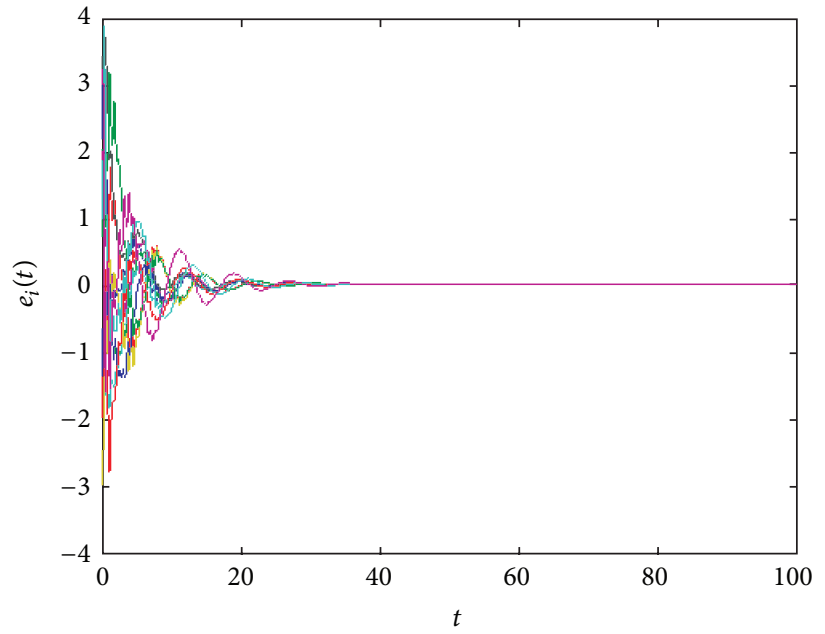

(b) $\mu=0.8$

FIGURE 4: Synchronization errors for the super network with different value of $\mu$. 
time and the coordinated scheduling in the process of transfer and optimization of the transfer facilities are of benefit to maintenance of the public traffic supernetwork. The urban decision-makers can adjust the departing frequency and optimization of the transfer facilities to improve the efficiency of the transfer and reduce waiting time of passengers during the rush hours.

\section{Appendix}

\section{The Proof on Theorem 4}

Consider the following Lyapunov candidate function:

$$
V(t)=\frac{1}{2} \sum_{i=1}^{N} e_{i}^{\mathrm{T}}(t) e_{i}(t) .
$$

Calculating its derivative along the trajectories of (A.1), under Assumption 2, we obtain

$$
\begin{aligned}
\dot{V}(t)= & \sum_{i=1}^{N} e_{i}^{\mathrm{T}}(t) e_{i}(t) \\
= & \sum_{i=1}^{N} e_{i}^{\mathrm{T}}(t)\left(g\left(y_{i}(t)\right)-g\left(x_{i}(t)\right)\right) \\
& -\sum_{i=1}^{N} e_{i}^{\mathrm{T}}(t)\left(\mu \sum_{j=1}^{N} c_{i j}+k\right) e_{i}(t) \\
& +\sum_{i=1}^{N} e_{i}^{\mathrm{T}}(t)\left(\varepsilon_{1} \sum_{j=1}^{N} a_{i j}+\varepsilon_{2} \sum_{j=1}^{N} b_{i j}-\mu \sum_{j=1}^{N} c_{i j}\right) e_{j}(t) \\
\leq & e^{\mathrm{T}}\left(L-\mu \sum_{j=1}^{N} c_{i j}-k+\varepsilon_{1} A+\varepsilon_{2} B-\mu C\right) e \\
\leq & e^{\mathrm{T}}\left(L-\mu c_{i}-k+\varepsilon_{1} A+\varepsilon_{2} B-\mu C\right) e,
\end{aligned}
$$

where $e=\left(e_{1}^{\mathrm{T}}, e_{2}^{\mathrm{T}}, \ldots, e_{N}^{\mathrm{T}}\right) \in R^{n N}, c_{i}=\min _{i} \sum_{j=1}^{N} c_{i j}$. Due to the matrix $A, B$ meeting the dissipative condition, there exists a large enough $k$ meeting condition (A.3) by adjusting the value of $\varepsilon_{1}, \varepsilon_{2}, \mu$,

$$
L-\mu c_{i}-k+\varepsilon_{1} A+\varepsilon_{2} B-\mu C \leq 0 .
$$

Namely, $\dot{V}(t) \leq 0$. According to the Lyapunov stability theory, we have $\lim _{t \rightarrow \infty} e_{i}(t)=0(i=1,2, \ldots, N)$. This completes the proof.

\section{Conflict of Interests}

The authors have declared that no conflict of interests exists.

\section{Acknowledgments}

The authors also gratefully acknowledge support from the National Natural Science Foundation of China (no. 61164003 and no. 61364001) and Lanzhou Jiaotong University Young Scientific Research Fund Project (no. 2014024), Government of China.

\section{References}

[1] C. Li, W. Sun, and J. Kurths, "Synchronization between two coupled complex networks," Physical Review E, vol. 76, no. 4, Article ID 046204, 2007.

[2] H. Tang, L. Chen, J.-A. Lu, and C. K. Tse, "Adaptive synchronization between two complex networks with nonidentical topological structures," Physica A: Statistical Mechanics and Its Applications, vol. 387, no. 22, pp. 5623-5630, 2008.

[3] J.-R. Chen, L.-C. Jiao, J.-S. Wu, and X.-H. Wang, "Adaptive synchronization between two different complex networks with time-varying delay coupling," Chinese Physics Letters, vol. 26, no. 6, Article ID 060505, 2009.

[4] M. Sun, C. Zeng, and L. Tian, "Linear generalized synchronization between two complex networks," Communications in Nonlinear Science and Numerical Simulation, vol. 15, no. 8, pp. 2162-2167, 2010.

[5] J.-A. Wang, H.-P. Liu, and X. Shi, "Adaptive synchronization between two different complex networks with time-varying delay coupling," Journal of University of Science and Technology Beijing, vol. 32, no. 10, pp. 1372-1378, 2010.

[6] Y. Sun, W. Li, and D. Zhao, "Outer synchronization between two complex dynamical networks with discontinuous coupling," Chaos, vol. 22, no. 4, Article ID 043125, 8 pages, 2012.

[7] Y.-J. Liu and S. C. Tong, "Adaptive fuzzy identification and control for a class of nonlinear pure-feedback MIMO systems with unknown dead zones," IEEE Transactions on Fuzzy Systems, vol. 23, no. 5, pp. 1387-1398, 2015.

[8] Y. J. Liu, Y. Gao, S. C. Tong et al., "Fuzzy approximation-based adaptive backstepping optimal control for a class of nonlinear discrete-time systems with dead-zone," IEEE Transactions on Fuzzy Systems, 2015

[9] Y. Sheffi, Urban Transportation Networks: Equilibrium Analysis with Mathematical Programming Methods, Printice-Hall, Upper Saddle River, NJ, USA, 1985.

[10] A. Nagurney and J. Dong, Supernetworks: Decision-Making for the Information Age, Edward Elgar Publishing, Cheltenham, UK, 2002.

[11] B. Wang, W. L. Wang, and X. H. Yang, "An optimal bus transport transfer algorithm based on weighted complex networks," Journal of Wuhan University of Technology, vol. 32, no. 6, pp. 1113-1116, 2008.

[12] W. Hui and H. Wang, "Empirical analysis of complex networks in public traffic networks," Computer Technology and Development, vol. 18, no. 11, pp. 217-222, 2008.

[13] J. J. Wu, Z. Y. Gao, H. J. Sun et al., Urban Traffic System Complexity-The Method of Complex Networks and Its Application, Science Press, Beijing, China, 2010.

[14] L. Zhao, M. Deng, J. Q. Wang, and D. L. Peng, "Structural property analysis of urban street networks based on complex network theory," Geography and Geo-Information Science, vol. 26, no. 5, pp. 11-15, 2010.

[15] Z. L. Hong, B. Y. Liu, and Y. P. Zhang, "Application of complex network in transportation network's node importance evaluation," Journal of Xian Technological University, vol. 34, no. 5, pp. 404-410, 2014. 
[16] X.-L. An, L. Zhang, and J.-G. Zhang, "Research on urban public traffic network with multi-weights based on single bus transfer junction," Physica A: Statistical Mechanics and Its Applications, vol. 436, pp. 748-755, 2015.

[17] Y.-J. Liu and S. C. Tong, "Adaptive NN tracking control of uncertain nonlinear discrete-time systems with nonaffine deadzone input," IEEE Transactions on Cybernetics, vol. 45, no. 3, pp. 497-505, 2015.

[18] Y. J. Liu, Y. Gao, S. C. Tong, and C. L. Chen, "A unified approach to adaptive neural control for nonlinear discrete-time systems with nonlinear dead-zone input," IEEE Transactions on Neural Networks and Learning Systems, vol. 27, no. 1, pp. 139-150, 2016.

[19] Y. Sun, W. G. Sun, and C. P. Li, "Synchronization analysis of two networks with mutual connections," Communication on Applied Mathematics and Computation, vol. 24, no. 2, pp. 49-54, 2010.

[20] H. K. Khalil and J. W. Grizzle, Nonlinear Systems, Prentice Hall, New Jersey, NJ, USA, 1996.

[21] J. S. Zhao, Z. R. Di, and D. H. Wang, "Empirical research on public transport network of Beijing," Complex Systems and Complexity Science, vol. 2, no. 2, pp. 45-48, 2005.

[22] M. Chang and S. F. Ma, "Empirical analysis for public transit networks in Chinese cities," Journal of Systems Engineering, vol. 22, pp. 412-418, 2007.

[23] Z. Y. Gao and Y. F. Song, "A reserve capacity model of optimal signal control with user-equilibrium route choice," Transportation Research Part B: Methodological, vol. 36, no. 4, pp. 313-323, 2002. 


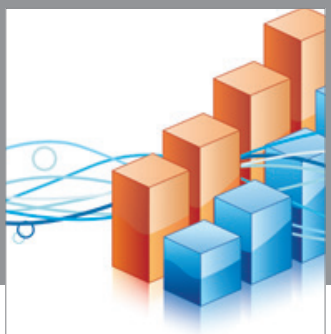

Advances in

Operations Research

vatem alat4

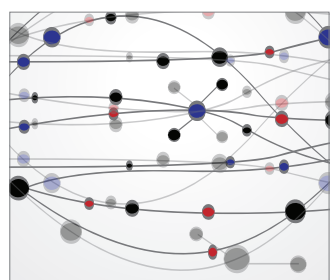

\section{The Scientific} World Journal
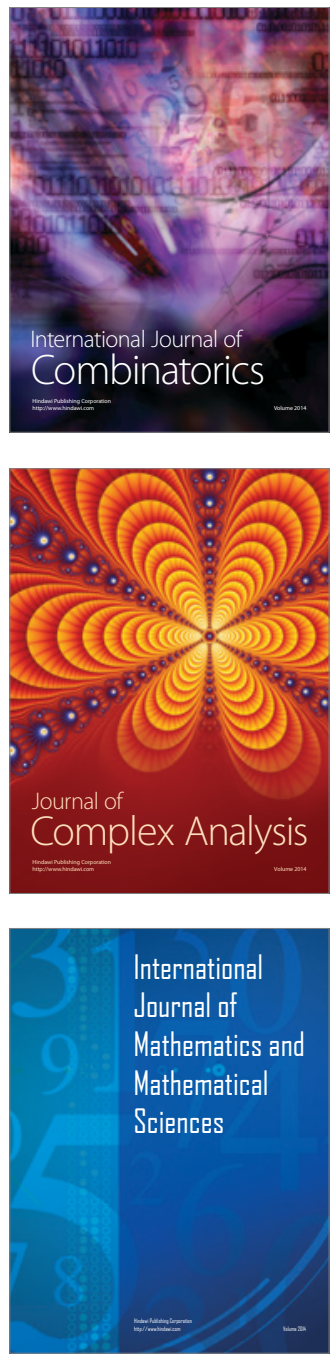
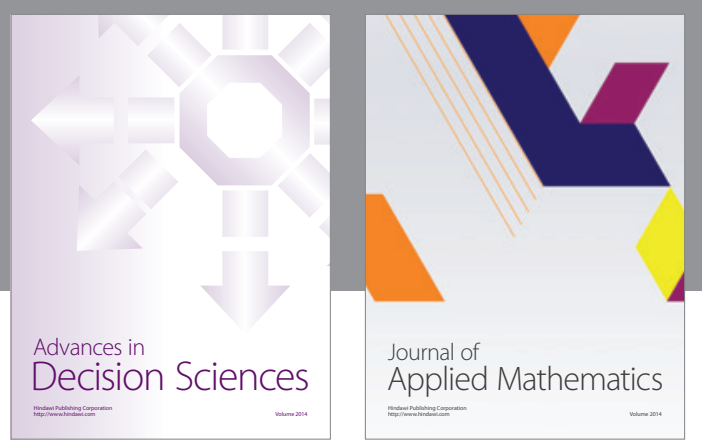

Algebra

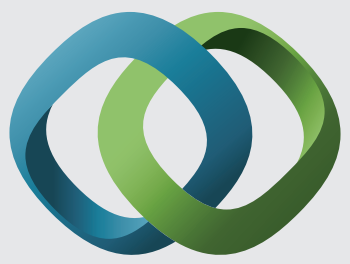

\section{Hindawi}

Submit your manuscripts at

http://www.hindawi.com
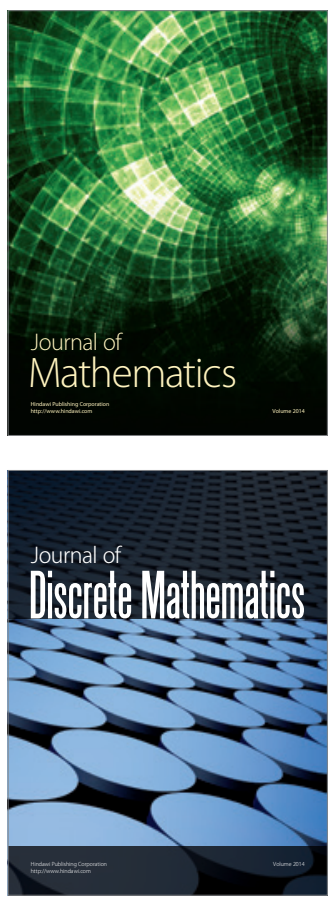

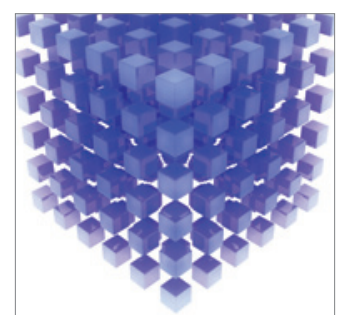

Mathematical Problems in Engineering
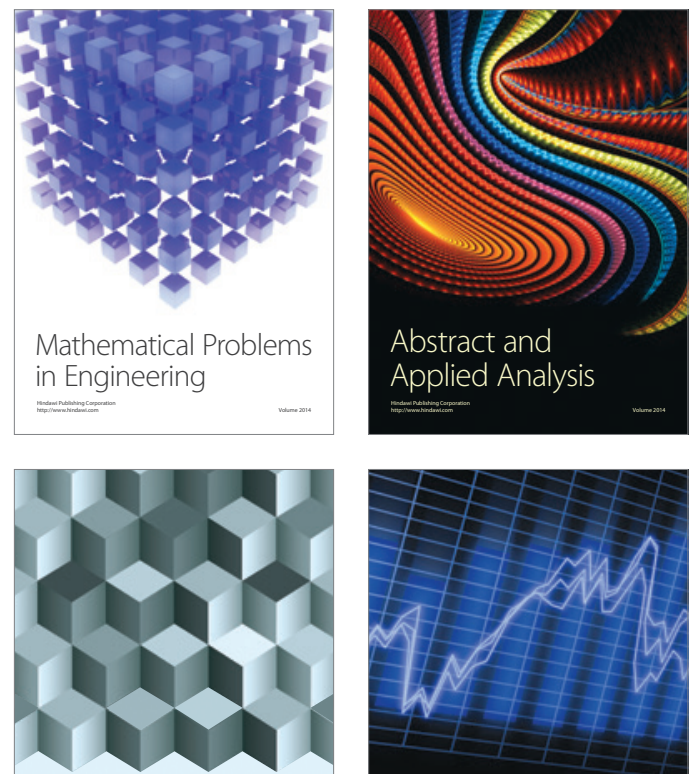

Journal of

Function Spaces

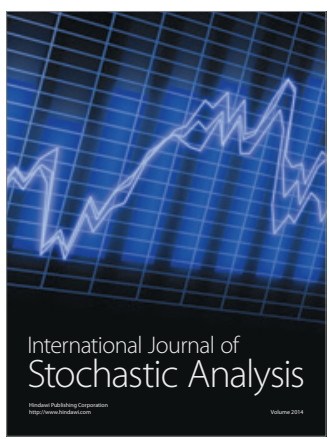

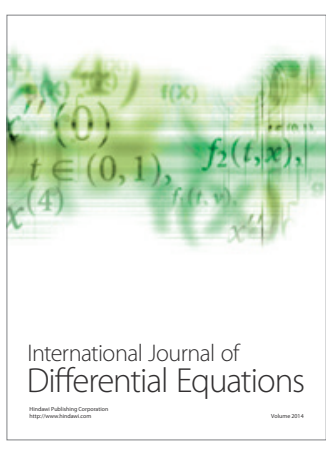
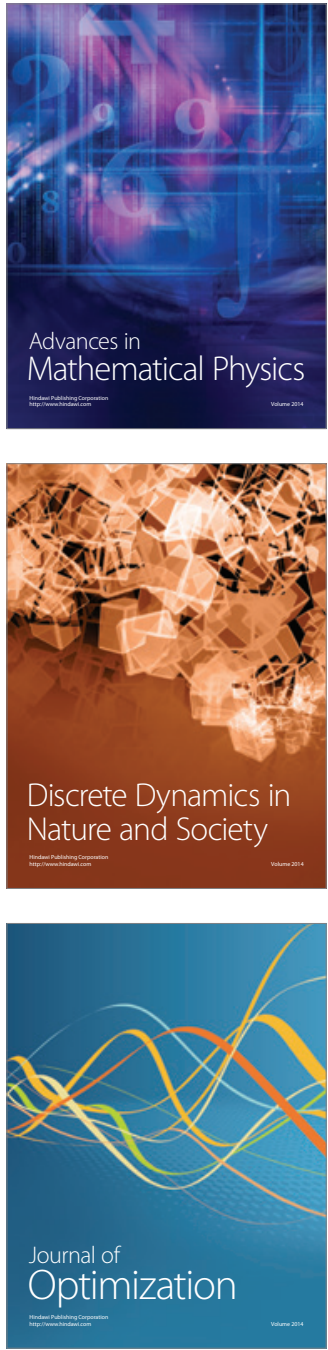